\title{
ACRF Archive User Meeting Summary
}

October 30, 2007

Reston, Virginia

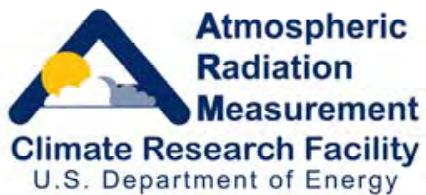

Work supported by the U.S. Department of Energy, Office of Science, Office of Biological and Environmental Research 


\section{DISCLAIMER}

This report was prepared as an account of work sponsored by the U.S. Government. Neither the United States nor any agency thereof, nor any of their employees, makes any warranty, express or implied, or assumes any legal liability or responsibility for the accuracy, completeness, or usefulness of any information, apparatus, product, or process disclosed, or represents that its use would not infringe privately owned rights. Reference herein to any specific commercial product, process, or service by trade name, trademark, manufacturer, or otherwise, does not necessarily constitute or imply its endorsement, recommendation, or favoring by the U.S. Government or any agency thereof. The views and opinions of authors expressed herein do not necessarily state or reflect those of the U.S. Government or any agency thereof. 


\section{Executive Summary}

On October 30, 2007, the U.S. Department of Energy's (DOE) Atmospheric Radiation Measurement (ARM) Climate Research Facility (ACRF) sponsored an all-day workshop to assess the status of the ACRF Archive. Focus areas included usability of current functions, plans for revised functions, proposals for new functions, and an overarching discussion of new ideas. Although 13 scientists familiar with ACRF and the ARM Program were invited to the workshop, only 10 scientists were available to attend the workshop.

ACRF consists of the infrastructure that was developed to support the ARM Program and includes the ACRF Archive (previously called the ARM Archive). The scientists who participated in the meeting ranged from those who used the Archive frequently to those who seldom or never had accessed the Archive. The group was spread across disciplines, i.e. modelers, conservationists, and others from universities and government laboratories. A few of the participants were funded by the ARM Program, but most were not funded currently by ARM.

During the past year, several improvements were made to the ACRF Archive to link it with the ARM/ACRF web pages, add a shopping cart feature, and expand on search parameters. Additional modifications have been proposed and prototypes of these proposals were made available for the participants. The participants were given several exercises to do before the meeting, and their feedback was requested to help identify potential problems and shortcomings with the existing structure and to recommend improvements.

The one-day meeting was structured along the following topics: (1) brief overviews of ACRF, ARCF data system, Archive history, evolution, and current status; (2) descriptions of recent Archive revisions and enhancements; (3) demonstration of a prototype of recent Archive developments; (4) descriptions of plans for future Archive revisions and enhancements; and (5) structured discussion of specific feedback questions. 


\section{Contents}

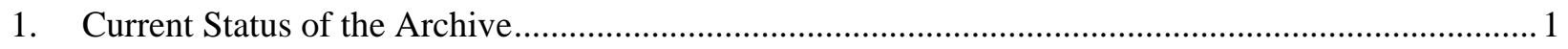

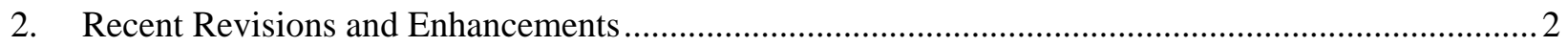

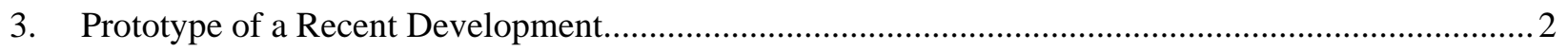

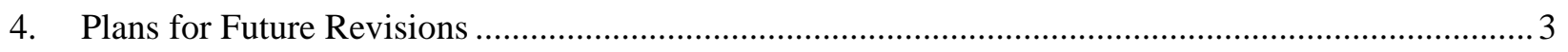

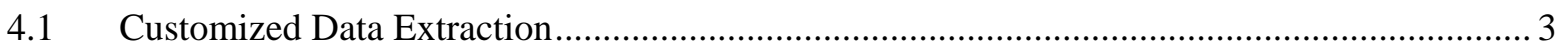

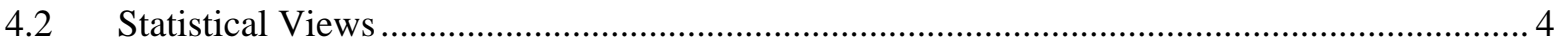

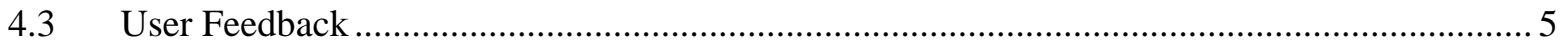

4.4 Revised Archive Login Strategy ................................................................................... 5

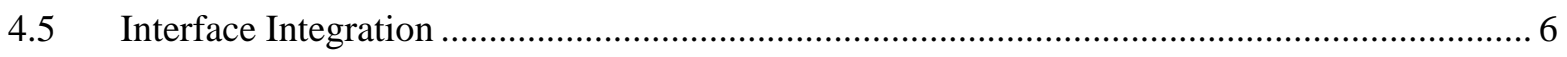

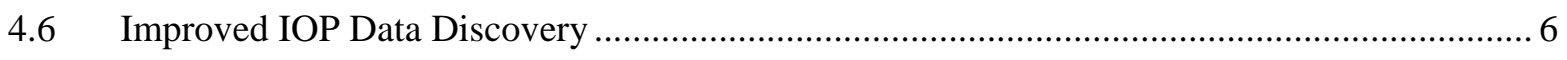

5. Workshop Participant User Feedback ……................................................................................. 7

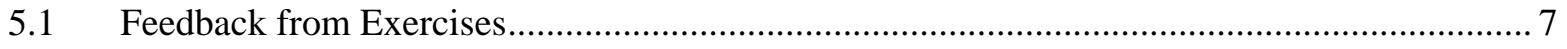

$5.2 \quad$ Participant Feedback During the Workshop....................................................................... 7

5.2.1 Primary Suggestions by Workshop Participant for Improving ACRF Archive ........... 7

Appendix A: List of Participants .................................................................................................. A.1

Appendix B: Feedback from User Group “Exercises” ....................................................................... B.1 


\section{Current Status of the Archive}

The Atmospheric Radiation Measurement (ARM) Program has been collecting a complex set of radiation, meteorological, and cloud data since 1992. These data are stored at, and distributed to users from, the ARM Climate Research Facility (ACRF) Archive at Oak Ridge National Laboratory (ORNL). This Archive is now part of the DOE user facility, known as the ACRF. Several different types of atmospheric and other data are available from the Archive. These run the gamut from continuously collected data using sensors at fixed-position ACRF sites to special data streams created by ARM principal investigators (PIs) to share with others in the ARM Program.

Continuous data are stored generally offline in an automated tape library and are accessible through requests from several user interfaces. These include ARM-collected data (e.g., radiation data from various sites), value-added products (VAPs; e.g., Active Remotely-Sensed Cloud Locations), and external data (e.g., National Oceanic and Atmospheric Administration [NOAA] satellites, model output). Special data, such as that from field campaigns and other PI-generated data are stored online and are directly accessible from a web interface. Raw unprocessed data files are available upon request but are not accessible from user interfaces. Processed data files are available from various user interfaces and are found in common data formats such as NetCDF and HDF.

VAPs are products from automated analytical procedures (e.g., models, retrievals, etc.) that are processed in the data system with inputs from instruments, other VAPs, and/or external data. The output of a VAP is a new ACRF data stream. VAPs include one or more of the following attributes: advanced algorithms; multiple data inputs; and long-term, continuous assessment of the quality of the input data. The ACRF produces some VAPs to improve the quality of existing measurements. In addition, when more than one measurement is available, the ACRF also produces "best estimate" VAPs.

External data in the Archive are generated by other programs (e.g., NOAA weather prediction models, NASA satellites, etc.) and are usually formatted into NetCDF, consistent with other ACRF files. These files include specialized subsets of interest to ACRF or specific to ACRF sites, such as geographic clips of global data. PI-generated data products from intensive operational periods (IOPs) are considered useful to ACRF users and are provided "at will” and supported by the researcher or owner of the data.

The scale of the Archive is large, containing data from many sites, from hundreds of sources and data users, and including thousands of measurements types. By December 2007, the Archive contained more than 118 trillion bytes of information in 8.3 million files. Researchers have requested more than 18 million files (89 trillion bytes) from routine data streams stored at the Archive. The Archive also has distributed (by way of user FTP access) more than 1.8 million files (1.7 trillion bytes) of IOP data. (IOP data sets are generally more concise than the ongoing ARM data streams.) The Archive has a cumulative "registered user base" of 3600 individuals. In a typical month, 100-120 users submit 400-600 requests for data.

Data can be accessed from the Archive using many different user interfaces. These user interfaces include the Web Shopping Cart, Data Browser, Thumbnail Browser, Catalog Browser, IOP Data Browser, NCVweb Data Plotting and Extraction Tool, and Telephone. There are continuous data distributions and "Standing Orders" for data from the Archive as well as on-time orders and requests for data files. 


\section{Recent Revisions and Enhancements}

One of the newest ways to search for and order ACRF data is the Shopping Cart feature on the www.arm.gov web pages. The Thumbnail Browser has been updated to allow the user to find data in much the same way as the Data Browser. After selecting data, the user can view thumbnail plots to gain insight into the data before requesting them. NCVweb has been made available as an additional interface to enable customized data extraction from files retrieved for data streams requested from the Archive, including the ability to plot data and calculate statistics "on the fly." The tabular displays of IOP information on the www.arm.gov website have been expanded to help the user search for data of interest by PI, year, site, and measurement type. When users who have established an account select an interface, they have a choice of entering their "user name" or their email address. This option should help users login more easily (infrequent use may make it harder to remember their unique Archive user name). In addition, this will help the Archive move toward consolidating accounts to one per person and simplify the reporting of usage statistics.

\section{Prototype of a Recent Development}

Although the participants completed pre-workshop exercises to evaluate many of the recent Archive developments (feedback is summarized in section 5), they worked through one of these developments during the workshop itself: the use of the Archive's Data Browser interface. The data browser is most useful when the user has predefined selection criteria (e.g., looking for data from a specific IOP or specific instrument at a site over a given period) and knows what he/she wants. Valuable comments were offered during this exercise, beginning with the login procedure.

There were several complaints about the login procedure, primarily concerning the extent of information requested from the user. The user is asked to establish an account at the Archive early in the process for searching for data files. This step required that the user supply detailed contact information for the purpose of compiling user facility statistics to report to DOE and the Office of Budget and Management. Several workshop participants felt that asking for address and phone number information over the web, for example, was a personal privacy intrusion making the user uncomfortable and could possibly deter some interested parties from continuing their search for data. It was suggested that this information (address and phone number) be made voluntary and that the login procedure be pushed further back into the session until the user has decided to begin an actual download. However, this discussion also led to the suggestion that the Archive make data plots simply viewable without logging in. If this enhancement can be made, then a less daunting login step early in the process for users actually intending to download data is not considered to be a problem.

Another issue regarding the data browser is associated with the choice given the user as to whether to use the "novice" or the "power" interface. These descriptors are not well defined on the web page. The "novice" interface was developed for users not already familiar with the ARM Program or the ACRF Archive. The "power" interface is for frequent users of the Archive and allows the user to skip many steps and go directly to the files of interest. It was suggested that the difference between the two be better defined and that new names should perhaps be given to the different interfaces. The term "novice" appears to imply an unintended association to some users (i.e., relating to their ability to use and understand the data, rather than their specific familiarity with ARM details such as the structure of data stream names). 
The participants were complimentary of the data plots that were available with some of the data. These plots were useful in helping them find the data they wanted and evaluate whether the use of other related data might be worthwhile. Several participants recommended an extended plotting capability for the Archive to help users elect data plot files for downloading. It was even suggested that Archive functionality be expanded to allow the user to perform multiple operations on various data and download only the results of these operations. This idea was discussed at length, as was the potential to develop a system similar to one at NOAA that allows users to manipulate selected data and store user data files on the system for retrieval at a later time. It was agreed that the potential for this development would require careful evaluation of related costs and implications on the Archive

\section{Plans for Future Revisions}

Continued improvement of the ACRF Archive is planned for the future. These improvements include the development of Customized Data Extraction functions, Statistical Views functions, solicitation methods for User Feedback, Revised Archive login strategies, Interface Integration methodologies, and Improved IOP Data Discovery.

\subsection{Customized Data Extraction}

This function will enable users to create customized data extractions from data files and from data-related to statistical summaries. User concerns related to the possibility of separating data from key metadata describing the measurement will be addressed. The data extraction function will be implemented in five increments as follows:

1. NCVweb will be modified to collect the specification of data extraction criteria from a files requested by a user. This function will enable data extraction for any portion of the ARM data that are stored in NetCDF files. This implementation is an interim solution to ensure a specific response is made to the fiscal year (FY) 2005 infrastructure review. This function should be enabled by May 2008.

2. An option to select specific measurements will be added to the measurements pathway of the Data Browser user interface. This will enable the extraction of selected measurements from large volumes of data from a single data stream without the "online” interaction required in NCVweb. The extracted data would be packaged in one (or more) files and the user notified by email when the output was ready for download. This function should be enabled by May 2008. This option and the one related to NCVweb will be evaluated for the inclusion of conditional data selection (criteria based on the ranges of selected measurements).

3. A data extraction function will be enabled for the data linked to the Statistical Views (see below). This function will only provide extraction capabilities for data described in Statistical Views. This function is one of several data access functions associated with Statistical Views. This function should be enabled by May 2008.

4. An extraction function will be enabled for a larger, more static (and online) collection of data. This is based on user feedback and experience with performance and capacity of the functions, as described in previous increments. This will be either an extension of the NCVweb function 
enabled for data requests or an extension of the extraction function enabled for Statistical Views with some "database" software. This functionality should be enabled by the end of FY 2008.

5. If needed (based on user feedback), the scope of data accessible for extraction and the performance of the extraction process will be improved during FY 2009. In addition, several participants suggested that it would be useful to extract integrated data files from the same period for multiple instruments. However, it was noted that the different sampling periods and potential offsets of the different instruments would make this much more extensive for data streams that are not already contemporaneous.

Other suggestions from the participants included the following:

- $\quad$ references to basic information and publications and a specific contact for each measurement

- $\quad$ better information and documentation about data quality and experimental uncertainties

- mentors or translators and a Wiki for each measurement or methodology as well as instrument

- $\quad$ automated messages, after file selection, suggesting related data files that may be of interest

- $\quad$ developing and updating of a library of “quality masks” for data files

- $\quad$ improving communication between those who discover problems in data files and the related instrument mentor.

Although these suggestions were considered important to maintaining a quality Archive, it was noted that these changes would need to be included in the list of growing requirements on the infrastructure should be considered in the context of all requested infrastructure support.

\subsection{Statistical Views}

This function will enable users to select and view statistical summaries of selected portions of Archive data. Current activities for statistical summaries are based on the Continuous Forcing (variational analysis) data product and the QCRAD (integrated radiation VAP data stream). The forcings data set is an example of a highly polished data set, specifically targeted for the modeling user community. QCRAD, also a highly polished data set, is an integrated radiation data set with a scope that fully spans ACRF operations (all sites and periods). It was selected as an example that would challenge the logic and performance of the Statistical Views concept. The "definition" of the statistical summaries will be developed by the translator with input from working group members. Actual summaries (graphs and statistical tabulation) will be generated either by the Archive or the translator (and other developer resources). The Archive will develop a web application that will allow users to select summaries to view. The ACRF web integration group will provide review and advice on the development of this application. 
The statistical summary application will provide access to the following four levels of information:

- the display of the graph from the statistics

- access to the statistical tabulation from the graph

- $\quad$ access to the data subset that was the basis of that summary (a form of data extraction)

- access to the data files that were the origin of the summarized data (an automatically generated data request). This function will include access to daily thumbnails, quicklooks, and Data Quality Reports (DQRs) typically associated with the data request process.

An application, as described above, will be completed by March 2008. The scope of the statistical views will expand in FY 2008 and FY 2009 as additional views are identified by the translators, feedback from users, and other requests. User feedback will be used to determine the value of dynamic, user-specified Statistical Views (probably during FY 2009). A near-term product expected to have Statistical Views is the Climate Modeling, Value-Added Best Estimate Product (a.k.a., the “Showcase Data set”), which is currently under development.

\subsection{User Feedback}

User feedback will be solicited in the following ways:

- By adding text boxes inside the user interfaces and selected web pages, the user can provide comments on interface problems, data discovery problems, descriptions of data usage, and other unmet user issues

- Identify a selection of Archive users (mostly non-ARM) to contact with specific follow-up questions about the above issues. The User Group meeting will be scheduled every two years, but the group may be reconvened for ad hoc meetings if detailed input is needed for significant changes.

\subsection{Revised Archive Login Strategy}

The login to the Archive will be modified in the following three ways:

1. The "username" will become the email address entered by the user (as it is needed to send the delivery notification). If the email address is not linked to a previous Archive user record, then detailed registration information will be requested. This change should enable the user to remember their "Archive ID."

2. The conditions under which a login is required will change to allow for a yet to be determined degree of anonymous graphical browsing (quicklooks and statistical views). Login will still be required once it is determined that a user actually wants to download data to their system.

3. A "single sign-on" will be enabled for all Archive (data ordering) applications. This revision is related to the interface integration described below. It will require some additional effort in FY 2009.

The first two modifications can be implemented during FY 2008. Implementing the "single sign-on” is likely to continue into FY 2009. 


\subsection{Interface Integration}

The integration of the Archive interfaces (Data Browser, Catalog Browser, Thumbnail, Web Shopping Cart, and IOP Data Browser) will include the following user functions:

- All interfaces can be accessed with a "single sign-on" (as referenced above)

- A single shopping cart will be shared by all interfaces. This will enable the user to take advantage of all available search strategies during a single session (if they prefer).

- The search parameters will be "shareable" between all interfaces. This will enable the user to change between the interfaces without the need to repeat the selection of basic search criteria such as site, date range, primary measurements, etc.

Interface integration work is not expected to begin until FY 2009 and is likely to extend beyond that year. The development of a truly integrated interface capability will take 1-2 years of effort and require a shifting of resource allocation priorities.

\subsection{Improved IOP Data Discovery}

The data discovery process for IOP data will be improved by the following changes:

- Replace the initial login prompt requiring the input of both a "username and password." The initial login will be limited to only the input of a username or email address for a previously registered user.

- Continue development of metadata for IOP data. This will enable the IOP web pages and summary tables on www.arm.gov to more directly guide users to the correct subdirectory.

- Review newer options for the style and technology of the IOP data browser.

- Solicit user comments on "points of confusion and suggested improvements" for the IOP data browser.

- Develop updates to the IOP data browser that address improvements in style, documentation and help information, and completeness of "readme" documentation. This will include the development of guidelines and a template for documentation so that more consistent information can be obtained during the submission of new data to the IOP system.

The improved logon will be implemented in FY 2008. The metadata development is ongoing (and should continue) by the web integration group. The review of the interface and solicitation of user comments should occur in the first quarter of FY 2008. The development of updates will occur in the remainder of FY 2008 and continuing into FY 2009, depending on the extent of the user feedback. 


\section{Workshop Participant User Feedback}

The workshop participants were given several exercises to do before the meeting and asked for feedback to help identify potential problems and shortcomings with the existing structure and to recommend improvements.

\subsection{Feedback from Exercises}

Most of the user group members provided feedback to the Archive via the exercises they were asked to complete before the meeting. This took considerable time and effort on their part and is much appreciated. This feedback was important because many of the user responses to the exercises served to frame the meeting discussion in advance.

Group members were sent two sets of exercises (with some Archive background and overview material): one on October 8 and the other on October 16. The first set consisted of searching and ordering exercises involving routine ARM data, using the shopping cart feature at www.arm.gov and the Data, Thumbnail, and Catalog Browser interfaces at the Archive. Users were given a choice between a scripted "novice" exercise and a more wide open and flexible "expert" exercise, in which they searched for several types of measurements using the different browsers. Both the novice and expert exercises received good attention from users. In the second set of exercises, users were asked to search for IOP data using the field campaign search page at ww.arm.gov and the Archive's IOP Browser. As a part of both exercises, users were asked to describe their experiences relating to the usability of the pages/interfaces. From this feedback, we learned what did and did not work well, where documentation and directions needed to be clearer, and what ideas users had for improvements. The exercises generated a lot of feedback that in many cases (especially related to finding IOP data) indicated the need for much improvement in the data searching/ordering process. Users carefully described issues and in some cases provided screen shots of the particular web pages where they found problems. Many of these details and experiences came up in the meeting and thus are included in the following section. Nonetheless, for completeness, a detailed summary of user responses to the exercises is included in Appendix B.

\subsection{Participant Feedback During the Workshop}

The workshop precipitated an active discussion between participants on needed and recommended improvements to the Archive. A summary of user suggestions is listed below. Where priority codes are given, they follow each suggestion in parentheses. The first letter is the priority class ( $\mathrm{HH}=$ very high, $\mathrm{H}$ = High, $\mathrm{M}=$ Medium, L=Low; and the second letter [for high priority items only] indicates level of effort and complexity: $\mathrm{H}=$ hard, $\mathrm{M}=$ moderate, $\mathrm{E}=$ easy). In some cases, no priority was assigned by the group. The suggestions below are divided into the following groupings: (1) "Word” changes, (2) Logic Changes, (3) Functional Revisions, (4) Functional Additions, and (5) Other.

\subsubsection{Primary Suggestions by Workshop Participant for Improving ACRF Archive}

\section{Word Changes}

- Make it clear that data are freely available to everybody at login (H E).

- Explain why login information is needed and make request for address and telephone optional. 
- Add/improve text on the create account process (L).

- Consider changing the wording of "create account" label. This may have "cost" connotations to some users $(\mathrm{H})$.

- Note total number of pages of the registration process (e.g. “ 1 of 2 ,” " 2 of 2,” etc.) on each page to $n$ indicate that the process is not "endless" $(\mathrm{H})$.

- Add “Access the Data Now?” at end of create account (change to create User ID) form (H).

- Functionally change the link to the Archive on ARM/ACRF website (H E).

- Rename “Order Data” button to "Build an Order” (H M).

- List instruments by what is recommended for each measurement, not alphabetically (HH).

- Add guidance to field campaign search form when "0 matches” result (e.g., add 1-800 number, link to Google IOP search (H).

- Rename data documentation category in field campaign navigation (H E).

- Change bullets to icons to indicate aircraft, satellite, ground, etc. (L).

- Show IOP vs. routine on measurement pages (H E).

- Show routine data that are really "measured" and which data are retrieved or modeled (M).

- Identify best estimates or recommended data stream for measurements/instrument web pages (HH $\mathrm{H})$.

\section{Logic Changes}

- Use the comparison of user interface options table as basis for navigating Archive.

- Create a web page that guides users on which interface to select and make this a prominent feature of the home page $(\mathrm{M})$.

- $\quad$ Expand pub searches/web of science for non-ARM Archive users; tell which data streams/sites/IOP data are used (M).

- Develop sorting by measurement type or VAP class, not instrument (H).

\section{Functional Revisions}

- Create a "browse/guest" account for new Archive users or defer this by creating more guidance on how to find data plots (L).

- $\quad$ Rename and better define the novice and power interfaces (or consider merging them) (M).

- Consider adding "static" information around the data stream pick list that explains how to decode the data stream name.

- Include access to display of "instrument class descriptions” (M).

- For those who choose not want to login, add link to data plots on the ARM/ACRF website. 
- Create search capability for accessing data streams by name; put in the view cart page.

- Enable the Archive to use qualitative analytical tools (coincidence results - tabular or graphical) as a means of providing a preview to the user.

- Enable web-based analysis and data extraction with quantitative output.

\section{Functional Additions}

- List a parameter scientist for each measurement who will take a leadership role and be the contact; refer to publications (HH).

- Consider adding more usable information in the " $i$ " in the thumbnail browser/power user interface, e.g., instrument name (H E).

- Include pop-ups or hyperlinks to descriptions of sites, facilities, and data streams, even on power user interface pages.

- $\quad$ Add a link on instruments page to papers published - ( $\mathrm{M}$ for all; $\mathrm{H}$ for one or two papers).

- Provide a web form to select data streams (instrument /VAP classes), measurements, sites (and maybe date ranges) used for a publication. Add this to the process used by ARM PIs. Add this as a requirement for renewal.

- Make DQRs “machine readable”; use a quality mask (H M).

- Develop conditional sampling within a single data stream; or for data extracted from multiple identical data streams $(\mathrm{H})$

- Send a yearly survey/questionnaire requesting feedback on Archive use and how the data are being used; create a graphic for web (M).

- Provide lists of previous Archive data users by data stream.

- Provide more information on quality control and experimental error in field campaign metadata and data types.

- Add data plots (with data) from PI in field campaigns (H E).

- Increase documentation for field campaigns; provide a standardized form for PIs to build readme files (M).

- Create link to readme information from field campaign data table (H M).

- Provide Estimate of Error/known uncertainties for all ACRF instruments.

- Encourage PI to submit PI plots to be included in IOP data sets, and provide a corresponding browser.

\section{Other}

- $\quad$ Finish metadata for the IOP system (H).

- Finish statistical summary views $(\mathrm{H})$. 
- Complete the revised functions of extracting measurements from a single data stream and packaging that extraction into a different format (single file vs. multiple files, NetCDF vs. comma delimited, etc.).

- Consider an OPeNDAP-type service for ARM and ACRF IOP data. This would enable using the Archive as one's hard drive without ordering and downloading data files.

- Consider incorporating Wikis into the system.

- Enable a web function to capture/display user comments or dialog (e.g., moderate blog or Wiki). 


\section{Appendix A: \\ List of Participants \\ ACRF User Group Meeting}

\section{ACRF User Group}

Jeff Amthor, DOE/OBER/Office of Science (not present at the meeting)

Roni Avissar, Duke University (not present at the meeting)

Larry Berg, PNNL

Ann Fridlind, NASA/Goddard Institute for Space Studies

Tom Jackson, U.S. Department of Agriculture (not present at the meeting)

Everette Joseph, Howard University

Rao Kotamarthi, ANL

Jay Mace, University of Utah

Sethu Raman, NC State

Kirstie Stramler, BNL

Dave Turner, University of Wisconsin

Hans Verlinde, Penn State University

Betsey Weatherhead, NOAA

\section{ACRF Infrastructure}

Sylvia Edgerton, PNNL

Rolanda Jundt, PNNL

Dale Kaiser, ORNL

Jim Mather, PNNL

Raymond McCord, ORNL

Sean Moore, Mission Research and Technical Services

Giri Palanisamy, ORNL

\section{DOE Program Manager}

Wanda Ferrell, OBER/Office of Science 


\title{
Appendix B:
}

\section{Feedback from User Group "Exercises"}

\section{(members were asked to complete in advance of the meeting)}

\section{Using Interfaces for Routine ARM Data}

\author{
Number of Respondents - 7 \\ Novice users -1 \\ Expert users -6
}

\section{- WWW.arm.gov}

- From several pages (e.g., a specific data stream page), seems unnatural to have "add to cart” before any site or date range information is specified.

- Instrument, Measurement, or Data tabs on www.arm.gov; not implicit that they all take you to the data (some confusion there). Comment "All the data are not under Data?"

- This is where some mentioned login problems.

- Looking for best estimate of cloud base at Barrow; know ARSCL is needed; it's not apparent how to access NSA ARSCL files using the links provided on the page. Lots of the links result in a closed loop.

\section{- Data Browser}

- On Archive home page, browser links within main text goes to documentation rather than the browser itself. Clarify what users should expect.

- For power user interface, at data stream selection level, ARM documentation links to "Data Stream." This link should probably be more descriptive, since it links to a page that shows the structure of data stream names. At that same point, a link to a page with explanations of trickier stuff, like the "data level" file extension would be useful. This type of convenient "legend" information could not be found in the browsing process. Archive comment: However, the power interface presumes familiarity and users explanation of terms should use the novice interface.

- A legend or link to data stream naming conventions on every screen where the data stream names are encountered would be helpful.

- For power user interface, a good feature is ability to choose only specific files/days within a date range for the final order.

- Because I have a Mac, Control-Click doesn't select non-adjacent data streams. I had to go in again to order nsaskyrad60s.C1.b1 - couldn't get them both at once!

- Love the quicklooks and that you can order across a year.

- Comparing two quicklooks on the same screen would be nice.

- This is my favorite access point into the Archive.

- Only able to select files to order from very long list. If I knew exactly which data stream I needed (files contained cloud base height). To do this, I need to know the details of the radar modes, the ARM processing of the modes, and then finally that the cloud base best estimate is hiding in the very big cdf file I'm about to download, of which only an infinitesimal fraction contains the data I'm interested in. If I don't know the operating 
modes, I'll download all five versions of the file, and then explore in them. Archive comment: Makes the case for data extraction capability.

- Strange "license / QC" truncated email received when data was ordered.

- It seems more difficult to order data from multiple instruments for the same period. In the past I have found that I must start over for each instrument of interest, reenter all of the dates, times, and locations, and then find the correct instrument. Archive comment: The user missed "select one or MORE...." in the menus.

- Browser should have a popup that shows what the abbreviation for each of the instrument stands for, and the measurements made, and the available date range. For example, when I highlight sgp15ebbrE7.a1, it would be useful to have a popup that tells me what the data are and other information.

\section{- Thumbnail Browser}

- Would be useful to be able to save and download thumbnail plots when one is viewing them.

- Why are there selection check boxes both at the top and bottom of thumbnail plots?

- Selected “all” for a week's worth of 2 displayed variables; clicked “add to shopping cart; next page that opened (http://www.archive.arm.gov/tnb/servlet/TNBController?service=ShoppingCart) was entirely blank and data were not ordered

- An interesting interface.

- There were no error messages, but the browser was unable to load http://www.archive.arm.gov/tnb/servlet/TNBController?service=ShoppingCart

- No data were found for the time period of interest for atmospheric profiling. Is this because there are no data, or because the images have not been created? A little more feedback to the user would be nice.

- It would be nice to include the time conversion from UTC to local standard time.

- It would be nice to include the time conversion from UTC to local standard time.

- $\quad$ Data browser showed the EBBR 15-minute data set. The Thumbnail browser had the 30minute data.

- The explanation of the data that are represented at the ' $\boldsymbol{i}$ ' button could be a little more informative.

\section{- Catalog Browser}

- Ability to order data files from individual days rather than just complete months would be useful. Archive Comment: Need to explain the computational background of this interface. Might be able to "rewire" the "select files function", but this is old software and interface needs update first.

- This is a good system for finding which data streams might be available, but it is difficult and time consuming to order data from multiple sites. It might be helpful to add a map or some site information to the page that lists the facilities. Archive Comment: User did not understand that you can click directly on sites link to change sites. Might want to emphasize on the start page "(Click on a non-zero cell in the table to choose a Site/Year and proceed to the next level of detail)" more, as users will click on the site on left, then have to go back to start the real drill down. Make font bigger, more pronounced. This applies to all the pages as one drills down. 
- At the instrument category page, clicking on a category on the left takes you to the shopping cart. If you proceed, then you have to enter data range, etc., and can get data.

- Easy to get off on an unintended path due to things being illogical with too many red herrings along the way. Archive note: if instructions for clicking on table entries are more prominent, this will be less likely to happen.

- This interface was better organized and had more information on the data sets right on the screen and less guessing as to what I would be getting.

\section{Using Interfaces for Field Campaign (IOP) Data}

Number of Respondents $\mathbf{- 4}$

\section{- IOP Browser}

- Folder names don't give clues as to which instruments/measurements are involved (e.g., at the /arm-iop/2003/sgp/aerosol/ level). The PI/institution names are of limited usefulness unless one knows countless associations.

- Recommend adding some of the advanced cross-referencing features of the www.arm.gov site, so that "guesses" relating to site/instrument/measurement don't need to be made as often.

- Login trickiness; "wasted time trying to figure out what you (the Archive) wanted".

- I could do any of the three exercises easily. Most of that is due to my familiarity with the IOPs, so I knew exactly who created which data sets and what was in them. I've always liked this interface. I've probably used it 10 times over the past several years.

- A link of some kind to the netCDF header would be helpful to show exactly what data are included in the file.

\section{- $\quad$ www.arm.gov Field Campaign Page}

- This page worked very well (using the search capability) for a user that had some previous knowledge of the Aerosol IOP.

- For individual IOP Participants/Data Sources that appear in IOP tables, it would be helpful to have a list of instruments that provide a particular measurement, like in the www.arm.gov pages for routine data.

- Surprised that Campaign Name link goes to a description of the experiment, not the data.

- Looking for MPACE data. I set the start and end dates for the start and end of the year (2004). I also tried it without the keyword search. When I did a similar search, but using only David Turner in the search window, I did find a number of data sets, but none were MPACE data sets.

- Screen shot of "NSA, Turner, AERI" - NO MATCHES.

\section{- Shopping Cart}

- After selecting an instrument, it’s non-intuitive having to "Add to Cart" before specifying the IOP of interest.

- This exercise was a waste of time. The instructions took way too much time and were not clear; was able to order data. 
- Primary weakness of IOP data searching is the large number of instrument categories that appear. They tend to reflect the way the measurement is made for example (AERI), rather than the quantity being measured (say temperature). If one is familiar with the data and the instruments, this is not a large problem, but if you are not, it requires more searching.

\section{Login and Interface Issues: Rankings of Importance by Users}

\section{Levels of Importance:}

(1) not important

(2) minimal importance

(3) somewhat important/helpful

(4) very important

(5) absolutely critical - must have it

How important is the ability to browse, search, and look at plots of ARM data without first having to login to the site?

Respondent 1: $\{(4)$ very important - because creating a user profile takes time that the casual data browser does not want to invest.

Respondent 2: \{(1) not important\}

Verbal responses: new users $-\{(4)$ very important $\}$; ARM experts $-\{(2)$ minimal importance\}

\section{What do you think/feel when you are presented with a login screen?}

Respondent 1: No problem if it seems necessary; don't like the idea of searches being tracked, on principle; hate user profile screens that have to be filled out before being allowed to login the first time.

Respondent 2: could scare away some folks when forcing people to login initially, especially if they are new users.

How important is communicating to the user as part of the Account Creation process the various reasons why the ACRF must ask them to create an account and login?

Respondent 1: $\{(4)$ very important $\}$

Respondent 2: $\{(4)$ very important $\}$ - Just the reason "we want to be able to send you updates on the data quality as it becomes better known" should be more than enough to warrant the tracking, in my opinion.

Respondent 3: \{(3) somewhat important/helpful $\}$ 
How important is moving away from the 3-frame display currently used in the IOP Browser and more fully extending the web-based searching of field campaigns found on the www.arm.gov field campaign page for IOP data discovery? (The IOP browser application would be maintained for packaging and ordering the IOP data.)

Respondent 1: $\{(4)$ very important $\}$

Respondent 2: \{(2) minimal importance\} - because I usually know exactly what I'm looking for. How well does this interface allow someone to select data from multiple IOPs before they hit "submit request"?

Respondent 3: \{(3) somewhat important/helpful\} - web-based field campaign searching seems a bit clumsy.

How important is the ability to seamlessly move between the various ARM data browsers (interface integration) without having to exit one browser, login to another, and then respecify basic parameters like site, facility, date range, measurement type, etc.?

Respondent 1: \{(3) somewhat important/helpful - fine with a more old-fashioned approach that gets at the same files three different ways without risk of accidentally ending up somewhere (in another browser) you didn't intend (or want) to go.

Respondent 2: \{(2) minimal importance $\}$ - would be a bonus though.

Respondent 3: \{(4) very important\}

How do you feel when you are presented with a login screen after you have already logged in?

Respondent 1: Accustomed to it.

Respondent 2: Frustrating; should only have to do it once.

Respondent 3: No problem.

Have you encountered the need to change between interfaces?

Respondent 1: yes

Respondent 2: no, because I go directly to the place I know will give me the data.

Respondent 3: Don't like having to reenter information when I need data from a subset of months over several years. For example, I have wanted to look at the TSI images for the summer-time months. Must go through many of the same steps numerous times.

If so, in what percentage of your data discovery sessions are you likely to change between user interfaces?

Respondent 1: $10 \%$

Respondent 2: 50\% 